\title{
Chebyshev solution of the nearly-singular one-dimensional Helmholtz equation and related singular perturbation equations: multiple scale series and the boundary layer rule-of-thumb
}

\author{
John P. Boyd \\ Department of Atmospheric, Oceanic and Space Science and Laboratory for Scientific Computation, \\ University of Michigan, 2455 Hayward Avenue, Ann Arbor, MI 48109, USA \\ E-mail: jpboyd@engin.umich.edu
}

Received 5 March 2003; accepted 12 November 2003

\begin{abstract}
The one-dimensional Helmholtz equation, $\varepsilon^{2} u_{x x}-u=f(x)$, arises in many applications, often as a component of three-dimensional fluids codes. Unfortunately, it is difficult to solve for $\varepsilon \ll 1$ because the homogeneous solutions are $\exp ( \pm x / \varepsilon)$, which have boundary layers of thickness $\mathrm{O}(1 / \varepsilon)$. By analyzing the asymptotic Chebyshev coefficients of exponentials, we rederive the Orszag-Israeli rule [16] that $N \approx 3 / \sqrt{\varepsilon}$ Chebyshev polynomials are needed to obtain an accuracy of $1 \%$ or better for the homogeneous solutions. (Interestingly, this is identical with the boundary layer rule-of-thumb in [5], which was derived for singular functions like $\tanh ([x-1] / \varepsilon)$.) Two strategies for small $\varepsilon$ are described. The first is the method of multiple scales, which is very general, and applies to variable coefficient differential equations, too. The second, when $f(x)$ is a polynomial, is to compute an exact particular integral of the Helmholtz equation as a polynomial of the same degree in the form of a Chebyshev series by solving triangular pentadiagonal systems. This can be combined with the analytic homogeneous solutions to synthesize the general solution. However, the multiple scales method is more efficient than the Chebyshev algorithm when $\varepsilon$ is very, very tiny.
\end{abstract}

Keywords: Chebyshev polynomials, spectral method, spectral element method

AMS subject classification: 42C10, 65M70, 65N35

\section{Introduction}

Through dimension-splitting, large semi-implicit hydrodynamics often must solve the one-dimensional Helmholtz equation,repeatedly at every timestep. If a fluid model is periodic in two dimensions, discretized by a Fourier spectral method with basis functions of the form $\exp (\mathrm{i} k y+\mathrm{i} m z)$, then to treat the viscous terms in the Navier-Stokes equations, it is necessary to solve the Helmholtz equation in the third dimension,

$$
\varepsilon^{2} u_{x x}-u=f(x),
$$


for each degree-of-freedom in the other two coordinates and at every time step - millions of one-dimensional Helmholtz solves in the course of the integration. The parameter $\varepsilon$ is

$$
\varepsilon=\frac{1}{\sqrt{k^{2}+m^{2}+2 /(v \delta t)}}
$$

where $v$ is the viscosity coefficient and $\delta$ is the timestep. For a high Reynolds number flow $(v \ll 1), \varepsilon$ is in the range of $1 / 10,000$ to $1 / 1000$ [7], [Diamessis and Domaradzki, submitted]. The homogeneous solutions to the Helmholtz equation $\operatorname{are} \exp ( \pm x / \varepsilon)$. If these boundary layers are not adequately resolved, the numerical solution will display spurious oscillations.

An obvious remedy is a change of coordinates so that an evenly spaced set of subdomains or grid points in the computational coordinate is equivalent to variable resolution with several grid points in the boundary layer itself. Whole books and reviews have been written on numerical resolution of boundary layer problems such as [13] and [12], and there have been plenty of articles in spectral methods realm on variable resolution/change-of-coordinate strategies including [10,11,14,17]. However, these strategies are unsatisfactory when the Helmholtz equations arise as part of a multidimensional hydrodynamics code.

First, the boundary layers are spurious because their width is controlled in part by the timestep, and therefore has nothing to do with the physics. Second, the "parent" multidimensional model has its own spacing of subdomains, grid points and so on, and it is obviously unpleasant to superimpose the complications of a change-of-coordinate just to resolve spurious boundary layers.

In this article, we describe two better alternatives. The multiple scales singular perturbation strategy can be combined with any discretization of derivatives, not merely spectral, and it can be applied to differential equations with variable coefficients, not merely the constant coefficient Helmholtz equation. It is, however, based on a (usually divergent) asymptotic expansion in $\varepsilon$, and therefore is inaccurate unless $\varepsilon$ is small.

The second strategy, which assumes that $f(x)$ is a polynomial or can be approximated by a polynomial, computes an exact particular integral through a very efficient sparse matrix/Chebyshev polynomial procedure. Although restricted to the Helmholtz equation only, the Chebyshev algorithm is (ignoring roundoff) of unlimited precision.

When $N$ is sufficiently large to explicitly resolve the boundary layers, neither of these strategies is needed. Orszag and Israeli derived a criterion for resolving boundary layers but merely quoted the relevant formulas without a detailed discussion. Because the boundary layer rule-of-thumb is so important, we offer a detailed derivation and analysis in the next section.

In section 3, we describe the multiple scales procedure. The rest of the article is focused on the Chebyshev polynomial strategy. 


\section{Asymptotic Chebyshev coefficients of the exponential functions}

The Chebyshev coefficients of $\exp (\rho[x-1])$ are Bessel functions [15]:

$$
\exp \{\rho[-1+x]\}=\sum_{n=0}^{\infty} b_{n} T_{n}(x)=\exp (-\rho)\left\{I_{0}(\rho)+2 \sum_{n=1}^{\infty} I_{n}(\rho) T_{n}(x)\right\} .
$$

The "uniform" asymptotic approximation to the Bessel functions [1] gives

$$
b_{n} \sim \exp (-\rho) \sqrt{\frac{2}{\pi n}} \frac{1}{\left(1+z^{2}\right)^{1 / 4}} \exp (n \chi(z))\left\{1+\mathrm{O}\left(\frac{1}{n}\right)\right\},
$$

where $z \equiv \rho / n$ and

$$
\begin{aligned}
\chi(z) & \equiv \sqrt{1+z^{2}}+\log \left(\frac{z}{1+\sqrt{1+z^{2}}}\right) \\
& \sim \begin{cases}1-\log (2)+\log (z)+\frac{1}{4} z^{2} \pm \cdots, & z \ll 1, \\
z-\frac{1}{2 z}+\frac{1}{24 z^{3}}-\frac{1}{80 z^{5}} \pm \cdots, & z \gg 1 .\end{cases}
\end{aligned}
$$

In the limit $n \rightarrow \infty(z \ll 1)$, this simplifies to

$$
b_{n} \sim \sqrt{\frac{2}{\pi n}} \exp \left(-\rho+n\left\{1+\log \left(\frac{\rho}{2}\right)\right\}-n \log (n)\right), \quad n \gg \rho .
$$

The boundary layer rule-of-thumb is derived by looking at the opposite limit of $z \gg 1$, that is, $n \ll \rho$, the bottom line of (5), which yields

$$
b_{n} \sim \sqrt{\frac{2}{\pi \rho}} \exp \left(-\frac{1}{2} \frac{n^{2}}{\rho}\right) \quad n \ll \rho .
$$

Figure 1 shows the contours of relative error of this approximation: as $\rho$ increases, the range of accuracy in $n$ increases.

One might suppose that the asymptotic Chebyshev coefficients of $\cos (\rho x)$ and $\exp \{\rho[-1+x]\}$ would be very similar. This is true for $n>\rho$ : the coefficients of both functions fall proportional to $\exp (-n \log (n))$. Figure 2 shows that this assumption is false for smaller $n$. The coefficients of $\exp \{\rho[-1+x]\}$ decrease monotonically: as a Gaussian in $n$ for $n<\rho$. In contrast, the coefficients of $\cos (\rho x)$ are $\mathrm{O}(1)$ and oscillatory until $n>\rho$. The quasi-sinusoidal rule-of-thumb in [5] is completely inappropriate for describing boundary layers.

Somewhat arbitrarily, we define the boundary layer to be resolved when the last retained coefficient, $b_{N}$, has been reduced to about $1 / 100$ of $b_{1}$. (Because the coefficients are falling as a Gaussian function of $n$ and because $\left|T_{n}(x)\right| \leqslant 1$ for all $n$ and all $x \in$ $[-1,1]$, one can show that the error in truncating after the $N$ th term is conservatively bounded by $b_{N}$.) 


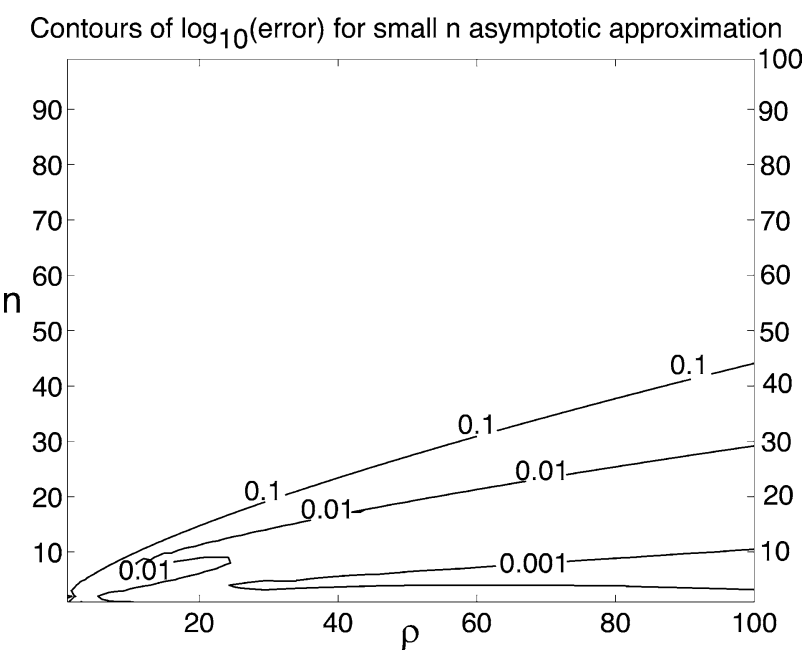

Figure 1. Contours of the base-10 logarithm of the relative errors, $\left|\left(b_{n}^{\text {exact }}-b_{n}^{\text {asymp }}\right) / b_{n}^{\text {exact }}\right|$ in the small- $n$ approximation, $b_{n} \sim \sqrt{2 /(\pi \rho)} \exp \left(-n^{2} /(2 \rho)\right)$, where the $b_{n}(\rho)$ are the Chebyshev coefficients of the function $\exp (\rho[x-1])$. The full asymptotic approximation (4) has a much wider range of accuracy (not shown).

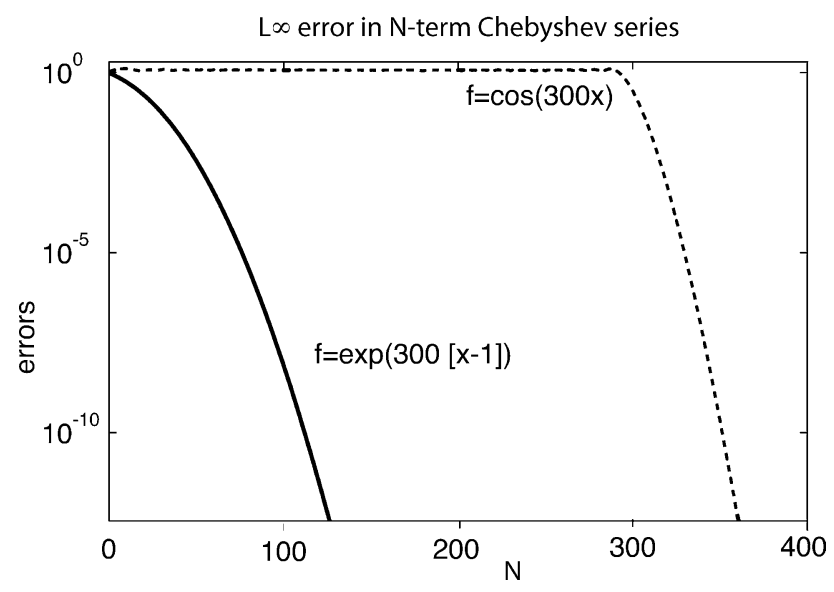

Figure 2. $L_{\infty}$ errors in truncating interpolated Chebyshev series after $N$ for $f(x)=\cos (300 x)$ and $f(x)=$ $\exp (300 x) / \exp (300)$. In the limit $\rho, N \rightarrow \infty$, the error curve for $\cos (\rho x)$ approaches a step function with the discontinuity at $N=\rho$.

In application to the homogeneous solutions of the Helmholtz equation, $\rho=1 / \varepsilon$. This gives the "boundary layer rule-of-thumb": to resolve a boundary layer of thickness $\varepsilon$, one must use a truncation $N$ of a Chebyshev polynomial basis that is sufficiently large so that

$$
N \geqslant 3 / \sqrt{\varepsilon} \quad \text { (boundary layer rule-of-thumb). }
$$




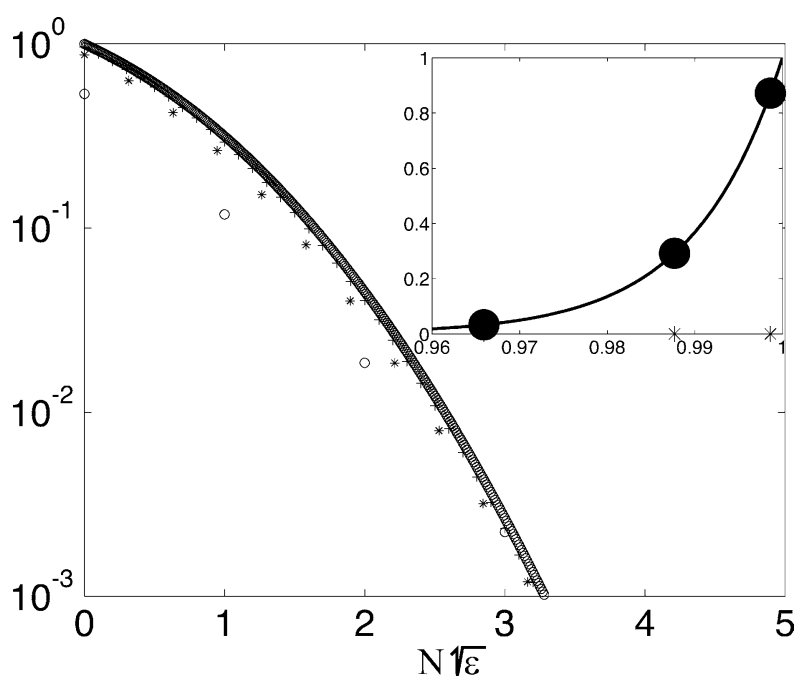

Figure 3. The plotted points in the main graph are the errors in the Chebyshev expansion of $f=\exp ([x-$ $1] / \varepsilon$ ) for various $N$ and $\varepsilon$. The graphs for different $\varepsilon$ were plotted using different $N$ scales so that the horizontal coordinate is $N \sqrt{\varepsilon}$ rather than $N$ itself. The curves fall almost on top of one another, confirming that the error is indeed a function of $N$ and $\varepsilon$ almost entirely through the combination $N \sqrt{\varepsilon}$. The e-folding scale of the inset figure is $1 / 100$; the black disks show the grid points for $N=30$, which is equivalent to $N=N_{\min }(\varepsilon)=3 / \sqrt{\varepsilon}$ for this value of $\varepsilon$.

Orszag and Israeli [16] quoted equation (7) and proposed (8) some thirty years ago. If $N>3 \varepsilon^{-1 / 2}$, then the multiple scales algorithm described in the next section is unnecessary.

The inset in figure 3, which illustrates the three grid points nearest the boundary for a typical small value of $\varepsilon$, visualizes equation (8). The $N \geqslant 3 / \sqrt{\varepsilon}$ criterion is identical with the rule-of-thumb stated in [5, p. 59 ], even though the latter is justified using the singular function $\tanh ([x-1] / \varepsilon)$, whereas the exponential is an entire function, free of singularities except at infinity. The universality of the $N \geqslant 3 \varepsilon^{-1 / 2} /$ two grid points-inthe-boundary layer criterion is rather pleasing.

\section{The method of multiple scales}

\subsection{One-dimensional Helmholtz equation}

Expanding $u(x ; \varepsilon)$ as a series in powers of $\varepsilon$ for the Helmholtz equation, $\varepsilon^{2} u_{x x}-$ $u=f(x)$, gives the particular integral

$$
\mathcal{P}=-\sum_{j=0}^{\infty} \varepsilon^{2 j} f_{2 j x}(x),
$$

where $f_{2 j x}$ denotes the $x$-derivative of order $2 j$. This solution is an example of the method of multiple scales [3, chapter 2] and [2]. The key assumption is that the particular 
integral is not a function of the "fast" variable, $x / \varepsilon$, but rather varies only on a "slow" $\mathrm{O}(1)$ length scale so that the second derivative of the particular integral is $\mathrm{O}\left(\varepsilon^{2}\right)$ smaller than the undifferential term. When $f$ is a polynomial, this series terminates and $\mathcal{P}$ must then be a polynomial, too. The general solution can be synthesized by adding arbitrary multiples of the homogeneous solutions, $\cosh (x / \varepsilon)$ and $\sinh (x / \varepsilon)$.

The series is an expansion in $\varepsilon^{2}$, not $\varepsilon$, so when $\varepsilon^{2} \sim \mathrm{O}\left(10^{-6}-10^{-8}\right)$ as common in applications, the two-term approximation

$$
\mathcal{P} \sim-f(x)-\varepsilon^{2} f_{x x}
$$

will be accurate to near machine precision!

The weakness of the multiple scales series is that when $\varepsilon$ is only moderately small, one must use many terms, which requires computing higher derivatives of $f$. It is well known that it is difficult to numerically differentiate functions to high order. Indeed, even when $f(x)$ is a polynomial, the size of the corrections becomes large and considerable ill-conditioning occurs (not illustrated). When $f$ is a function with singularities at any finite location in the complex plane, the multiple scales series diverges [3,4].

Thus, for moderate $\varepsilon$, such as $\varepsilon \approx 1 / 5$, the Chebyshev particular integral strategy is the only option. For very small $\varepsilon$, though, the multiple scales series is better.

\subsection{Generalization}

The multiple scales algorithm can be extended to variable coefficient equations such as

$$
\varepsilon^{2} u_{x x}-q(x) u=f(x),
$$

where $q(x)>0$.

When $\varepsilon \ll 1$ and Chebyshev expansion of the homogeneous solutions is too expensive, the WKB approximation [2] is very accurate:

$$
\begin{aligned}
& u_{1, \mathrm{hom}}(x) \sim \frac{1}{q^{1 / 4}} \cosh \left(\int^{x} \frac{\sqrt{q(y)}}{\varepsilon} \mathrm{d} y\right), \\
& u_{2, \mathrm{hom}}(x) \sim \frac{1}{q^{1 / 4}} \sinh \left(\int^{x} \frac{\sqrt{q(y)}}{\varepsilon} \mathrm{d} y\right) .
\end{aligned}
$$

Higher order WKB terms can be calculated [2] to obtain extremely high accuracy for $\varepsilon \ll 1$, even though the WKB series is usually divergent.

Similarly, a particular integral $\mathcal{P}$ can be generated by the method of multiple scales [3, chapter 2] and [2]. Assume

$$
\mathcal{P}=\sum_{j=0}^{\infty} \varepsilon^{2 j} u^{(2 j)}(x),
$$


where

$$
u^{(0)}=-\frac{f(x)}{q(x)}
$$

and where higher order terms are

$$
u^{(2 j+2)}(x)=\frac{u_{x x}^{(2 j)}}{q(x)} .
$$

The necessary derivatives can be computed to spectral accuracy by using Chebyshev interpolation [5].

\subsection{Uniformity in $\varepsilon$}

The road to a robust spectral method, uniformly accurate in $\varepsilon$, is to test-and-switch. First, choose the Chebyshev truncation $N$ and let $q_{b}$ denote the largest values of $q$ in the boundary layers. (The boundary layers will then have a thickness no smaller than $\varepsilon / \sqrt{q_{b}}$.) If

$$
\varepsilon>\frac{9 \sqrt{q_{b}}}{N^{2}} \equiv \varepsilon_{\text {switch }}
$$

then the boundary layers can be explicitly resolved, and one applies Chebyshev methods as in [5] without the need to treat the homogeneous and particular solutions individually. If $\varepsilon<\varepsilon_{\text {switch }}$, apply the WKB method and the multiple scales series, or combine the analytic homogeneous solutions for the Helmholtz equation with the Chebyshev particular integral strategy explained in the next section.

\section{Chebyshev series computation of an exact particular integral}

\subsection{Rescaling and simplification}

Galerkin and pseudospectral Chebyshev discretizations of the Helmholtz equation yield dense matrices which are relatively expensive to factor and solve. Clenshaw [6] showed nearly a half a century ago that one could obtain a sparse Chebyshev discretization by formally integrating the equation twice to transform it to

$$
\varepsilon^{2} u-\iint u=\iint f
$$

and twice applying the identity

$$
\int T_{n}(x) \mathrm{d} x=\frac{1}{2}\left\{\frac{T_{n+1}(x)}{n+1}-\frac{T_{n-1}(x)}{n-1}\right\}, \quad n \geqslant 2 .
$$




\subsection{Parity}

As explained at greater length in [5, chapter 8], an arbitrary function $f(x)$ can always be decomposed into its parts which are symmetric and antisymmetric with respect to the origin. The symmetric or "even parity" part is $f_{S}=(1 / 2)(f(x)+f(-x))$ and is symmetric in the sense that $f_{S}(-x)=f_{S}(x)$. The odd part is $f_{A}=(1 / 2)[f(x)-f(-x)]$ which is antisymmetric in the sense that $f_{A}(-x)=-f_{A}(x)$.

By similarly decomposing the solution into $u_{S}$ and $u_{A}$, the Helmholtz problem can be reduced to the uncoupled pair of equations

$$
\varepsilon^{2} u_{S}-\iint u_{S}=\iint f_{S}, \quad \varepsilon^{2} u_{A}-\iint u_{A}=\iint f_{A} .
$$

The even degree Chebyshev polynomials $T_{2 n}$ are symmetric while the $T_{2 n-1}$ are antisymmetric. Furthermore, the exponentials which are homogeneous solutions of the Helmholtz equation can be rearranged into hyperbolic functions of definite symmetry. Thus,

$$
u_{S}=A \cosh (x / \varepsilon)+\sum_{n=0}^{\infty} p_{2 n} T_{2 n}(x), \quad f_{S}=\sum_{n=0}^{\infty} f_{2 n} T_{2 n}(x)
$$

and similarly for the antisymmetric problem. By exploiting symmetry, one can reduce the bandwidth of the discretization matrices (by forming two matrices of half the size and smaller bandwidth) and thereby reduce the cost.

\subsection{Galerkin/tau method}

When the usual Chebyshev-Galerkin method (called the "tau method" in [9]) is applied to each of the two twice-integrated subproblems of definite symmetry, the result is a pair of nearly-tridiagonal matrices, one for the even degee coefficients $\left(a_{0}, a_{2}, a_{4}, \ldots\right)$ of $u$ and the other for the odd degree coefficients [5,8,9]. Introducing the auxiliary array $c_{n}$ where $c_{0}=2$, all other $c_{n}=1$, the condition that the inner product of $T_{n}(x)$ with the residual of the twice-integrated Helmholtz equation is zero gives

$$
\begin{gathered}
\varepsilon^{2} a_{n}-\frac{1}{4 n(n-1)} c_{n-2} a_{n-2}+\frac{1}{2\left(n^{2}-1\right)} a_{n}-\frac{1}{4 n(n+1)} a_{n+2} \\
=\frac{1}{4 n(n-1)} c_{n-2} f_{n-2}-\frac{1}{2\left(n^{2}-1\right)} f_{n}+\frac{1}{4 n(n+1)} f_{n+2},
\end{gathered}
$$

where the formula generates rows of both the even and odd problems, depending on whether $n$ is even or odd. ${ }^{1}$ It is understood that terms in $f_{n}$ and $a_{n}$ with $n>N$, where $N$ is the truncation of the Chebyshev series, will be omitted from these equations. The $n=0$ and $n=1$ cases are replaced by the boundary conditions when the whole solution is approximated by a truncated Chebyshev series.

\footnotetext{
${ }^{1}$ Note that (15.88) [5, p. 313] gives this formula with some sign errors.
} 
If $f(x)$ is a polynomial of degree $N$, the general solution is

$$
u(x)=A \cosh \left(\frac{x}{\varepsilon}\right)+B \sinh \left(\frac{x}{\varepsilon}\right)+\mathcal{P}(x),
$$

where $A$ and $B$ are the arbitrary constants multiplying the homogeneous solutions (to be determined by imposing the boundary conditions) and $\mathcal{P}$ is a particular integral that is also a polynomial of degree $N$ :

$$
\mathcal{P}=\sum_{n=0}^{N} p_{n} T_{n}(x) .
$$

Because $\mathcal{P}$ is computed as the sum of two separate series, it is convenient to write the homogeneous solutions so that each has definite symmetry with respect to the origin also. (Note that $\cosh (x / \varepsilon)=\cosh (-x / \varepsilon)$ whereas $\sinh (x / \varepsilon)=-\sinh (-x / \varepsilon)$.)

The same sparse equations still apply with $a_{n}$ replaced by $p_{n}$. However, because no boundary conditions are imposed on the particular integral, it follows that (i) the inner products with $T_{2}$ (for the even degree problem) and $T_{3}$ (for the odd degree problem) are now the first rows of the symmetric and antisymmetric problems, respectively, and (ii) we must also demand that the doubly-integrated differential equation should be orthogonal to $T_{N+1}$ and $T_{N+2}$, instead of only orthogonal to $T_{N}$ and polynomials of lower degree.

The symmetric subproblem, truncated after the fifth symmetric term, is

$$
\left|\begin{array}{ccccc}
-\frac{1}{4} & \varepsilon^{2}+\frac{1}{6} & -\frac{1}{24} & 0 & 0 \\
0 & -\frac{1}{48} & \varepsilon^{2}+\frac{1}{30} & -\frac{1}{80} & 0 \\
0 & 0 & -\frac{1}{120} & \varepsilon^{2}+\frac{1}{70} & -\frac{1}{168} \\
0 & 0 & 0 & -\frac{1}{224} & \varepsilon^{2}+\frac{1}{126} \\
0 & 0 & 0 & 0 & -\frac{1}{360}
\end{array}\right|\left|\begin{array}{c}
p_{0} \\
p_{2} \\
p_{4} \\
p_{6} \\
p_{8}
\end{array}\right|=\left|\begin{array}{c}
\frac{1}{4} f_{0}-\frac{1}{6} f_{2}+\frac{1}{24} f_{4} \\
\frac{1}{48} f_{2}-\frac{1}{30} f_{4}+\frac{1}{80} f_{6} \\
\frac{1}{120} f_{4}-\frac{1}{70} f_{6}+\frac{1}{168} f_{8} \\
\frac{1}{224} f_{6}-\frac{1}{126} f_{8} \\
\frac{1}{360} f_{8}
\end{array}\right|
$$

We see that the matrix is in upper triangular/pentadiagonal form and may be solved by Gaussian elimination (Cholesky factorization) in $\mathrm{O}(N)$ operations. The antisymmetric subproblem is of similar pentadiagonal/triangular form. As long as $f(x)$ is a polynomial of degree $N$ or less, (22) is an exact solution. The sole role of the auxiliary array $c_{n}$ is to multiply the coefficients of $p_{0}$ and $f_{0}$ by a factor of two in the first row of the matrix.

\section{The particular integral as a filter for spurious boundary layers}

When the parameter $\varepsilon$ is controlled by the timestep, the boundary layers have a thickness which is not controlled by the physics, but only by the time-marching algo- 
rithm. If the homogeneous solutions with their $\mathrm{O}(\varepsilon)$-thick unphysical boundary layers are included in the Helmholtz solution, then this spurious narrow length scale will be carried through later timesteps. The forcing function $f(x)$ will then vary on an $\mathrm{O}(\varepsilon)$ length scale and the spectral algorithm will fail unless $N>3 / \sqrt{\varepsilon}$, which is likely to be prohibitively large.

It follows that the homogeneous solutions must be ignored when $\varepsilon$ is controlled by the timestep. Both the multiple scale and Chebyshev particular integral strategies have the virtue of generating a particular integral which varies only on the slow scale of the forcing function, free of spurious boundary layers. To approximate $u(x)$ by the particular integral $\mathcal{P}$ is to filter the boundary layers contained in the homogeneous solutions.

\section{Summary}

We have shown that for the one-dimensional Helmholtz equation, $\varepsilon^{2} u_{x x}-u=f$, it is possible to obtain a spectral discretization which is uniformly accurate as $\varepsilon \rightarrow 0$ by computing a particular solution which depends only on the smoothness of the forcing, not the boundary layers of the homogeneous solutions. We have presented two strategies for generating such a slowly-varying particular integeral: the method of multiple scales and a Chebyshev-Galerkin algorithm.

When the width of these boundary layers is controlled by the timestep and these layers are spurious, they can be filtered out simply by approximating $u(x)$ by the particular integral. When $\varepsilon$ is independent of numerical parameters and the boundary layers are physical, the general solution can be obtained by adding in the homogeneous solutions so that the boundary conditions are satisfied. The homogeneous solutions can be generated analytically as hyperbolic functions (for the Helmholtz equation) and by WKB approximations for variable coefficient equations. In all cases, it is unnecessary to explicitly resolve the boundary layers through Chebyshev polynomial series.

By using the identities $\int^{x} P_{n}(y) \mathrm{d} y=\left(P_{n+1}(x)-P_{n-1}(x)\right) /(2 n+1), n \geqslant 1$ and $\int_{x} P_{0} \mathrm{~d} y=P_{1}$, one can easily derive a similar Legendre-Galerkin algorithm to compute the particular integral when $f(x)$ is a polynomial. We leave the details to the reader because there is no computational advantage even when the Helmholtz-Chebyshev routine is embedded in a Legendre spectral element code. The reason is that when the number of degrees of freedom per subdomain is moderate, all transformations from grid point values to Chebyshev or Legendre coefficients must be done by a matrix-vector multiply where the transformation matrix needs only to be computed once and stored, and likewise for the inverse transformation. The matrix that takes values of $f(x)$ at the Legendre-Lobatto points to Chebyshev coefficients is slightly different from that which yields the Legendre coefficients of $f$, but both matrix multiplications are of identical cost.

We have not presented numerical examples of the Chebyshev algorithm to compute a polynomial particular integral because the resulting Helmholtz solution is exact. We have not offered numerical examples of the accuracy and limitations of the method of multiple scales either since a full discussion is given in the books [2,3] and review [4]. 
Nevertheless, it would be interesting to see these ideas applied in the context of threedimensional hydrodynamics code and other demanding applications, but this is beyond the scope of this note.

\section{Acknowledgements}

This work was supported by NSF grant OCE9986368. I thank Peter Diamessis and J. Andrezj Domaradzki for suggesting this problem.

\section{References}

[1] M. Abramowitz and I.A. Stegun, Handbook of Mathematical Functions (Dover, New York, 1965).

[2] C.M. Bender and S.A. Orszag, Advanced Mathematical Methods for Scientists and Engineers (McGraw-Hill, New York, 1978) p. 594.

[3] J.P. Boyd, Weakly Nonlocal Solitary Waves and Beyond-All-Orders Asymptotics: Generalized Solitons and Hyperasymptotic Perturbation Theory, Mathematics and Its Applications, Vol. 442 (Kluwer, Amsterdam, 1998) p. 608.

[4] J.P. Boyd, The devil's invention: Asymptotics, superasymptotics and hyperasymptotics, Acta Applicandae 56 (1999) 1-98.

[5] J.P. Boyd, Chebyshev and Fourier Spectral Methods, 2nd ed. (Dover, Mineola, NY, 2001) p. 665.

[6] C.W. Clenshaw, The numerical solution of linear differential equations in Chebyshev series, Proc. Cambridge Philos. Soc. 53 (1957) 134-149.

[7] J.A. Domaradzki, An analytic Green's functions method in pseudo-spectral Navier-Stokes solvers for boundary layers and channel flows, J. Comput. Phys. 88 (1990) 232-242.

[8] L. Fox and I.B. Parker, Chebyshev Polynomials in Numerical Analysis (Oxford Univ. Press, London, 1968).

[9] D. Gottlieb and S.A. Orszag, Numerical Analysis of Spectral Methods (SIAM, Philadelphia, PA, 1977) p. 200.

[10] W. Heinrichs, Spectral methods for singular perturbation problems, Appl. Math. 39 (1994) 161-188.

[11] M.A.K. Ibrahim and R.S. Temsah, Spectral methods for some singularly perturbed problems with initial and boundary layers, Internat. J. Comput. Math. 25 (1988) 33-48.

[12] M.K. Kadalbajoo and K.C. Patidar, A survey of numerical techniques for solving singularly perturbed ordinary differential equations, Appl. Math. Comput. 130 (2002) 457-510.

[13] V.D. Liseikin, Layer Resolving Grids and Transformations for Singular Perturbation Problems (VSP/Brill/Academic Press, Leiden, the Netherlands, 2001).

[14] W. Liu and T. Tang, Error analysis for a Galerkin-spectral method with coordinate transformation for solving singularly perturbed problems, Appl. Numer. Math. 38 (2001) 315-345.

[15] Y.L. Luke, The Special Functions and Their Approximations, Vols. I and II (Academic Press, New York, 1969).

[16] S.A. Orszag and M. Israeli, Numerical simulation of incompressible flow, Ann. Rev. Fluid Mech. 6 (1974) 281-318.

[17] T. Tang and M.R. Trummer, Boundary layer resolving pseudospectral methods for singular perturbation problems, SIAM J. Sci. Comput. 17 (1995) 430-438. 